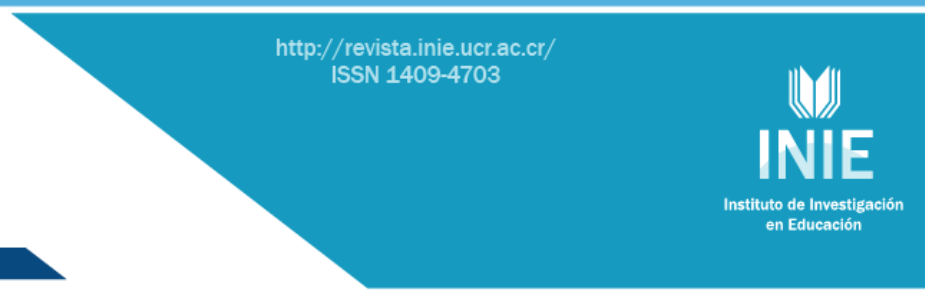

\title{
LINEAMIENTOS CURRICULARES, DESDE UNA PEDAGOGÍA CRÍTICA, PARA LA SELECCIÓN Y ORGANIZACIÓN DE LOS CONTENIDOS EN LOS PROGRAMAS DE ESTUDIO DEL MINISTERIO DE EDUCACIÓN PÚBLICA \\ CURRICULAR LINEAMENTS FROM A CRITICAL PEDAGOGY FOR THE SELECTION AND ORGANIZATION OF THE PROGRAMS' CONTENTS OF THE COSTA RICAN MINISTERY \\ OF PUBLIC EDUCATION
}

Volumen 15, Número 2

Mayo - Agosto

pp. 1-23

Este número se publicó el $1^{\circ}$ de mayo de 2015

DOI: http://dx.doi.org/10.15517/aie.v15i2.18971

César Toruño Arguedas

Revista indizada en REDALYC, $\underline{\text { SCIELO }}$

Revista distribuida en las bases de datos:

CATÁLOGO DE LATINDEX, IRESIE, CLASE, DIALNET, DOAJ, E-REVIST@S, SHERPA/ROMEO, QUALIS, MIAR

Revista registrada en los directorios:

ULRICH'S, $\underline{\text { REDIE}}, \underline{\text { RINACE}}, \underline{\text { OEI }}, \underline{\text { MAESTROTECA }}, \underline{\text { PREAL, }} \underline{\text { CLACSO }}$ 


\section{LINEAMIENTOS CURRICULARES, DESDE UNA PEDAGOGÍA CRÍTICA, PARA LA SELECCIÓN Y ORGANIZACIÓN DE LOS CONTENIDOS EN LOS PROGRAMAS DE ESTUDIO DEL MINISTERIO DE EDUCACIÓN PÚBLICA \\ CURRICULAR LINEAMENTS FROM A CRITICAL PEDAGOGY FOR THE SELECTION AND ORGANIZATION OF THE PROGRAMS' CONTENTS OF THE COSTA RICAN MINISTERY \\ OF PUBLIC EDUCATION}

César Toruño Arguedas ${ }^{1}$

Resumen: El ensayo plantea una propuesta de criterios curriculares para la selección y organización de contenidos de los programas de estudio de las diferentes asignaturas del Ministerio de Educación Pública, para lo cual establece una fundamentación teórica y su posible aplicación en la cotidianidad de las comisiones de revisión y reforma de los programas de estudio. Además, se establecerán elementos para la incorporación de principios de la pedagogía crítica en el proceso curricular, todo lo cual pretende establecer viabilidad de la estructuración del proceso curricular y la inclusión de la pedagogía crítica en el quehacer del Ministerio de Educación Pública, Costa Rica.

Palabras clave: CURRÍCULUM, PEDAGOGÍA CRÍTICA, SELECCIÓN Y ORGANIZACIÓN DE CONTENIDOS, PROGRAMAS DE ESTUDIO, MINISTERIO DE EDUCACIÓN PÚBLICA, COSTA RICA

Abstract: This essay has the goal to propose curricular criteria for the selection and organization of the programs' contents of the different subjects of the Costa Rican Ministry of Public Education. To reach this, it is established a theoretical foundation to apply to the Review commissions and programs daily. Furthermore, we establish elements for the incorporation of principles of the critical pedagogy into the curricular process in order to give practicable to the whole curricular process and including the critical pedagogy in the Ministry of Public Education's processes.

Key words: CURRICULUM, CRITICAL PEDAGOGY, SELECTION AND CONTENT ORGANIZATION, PROGRAMS, COSTA RICAN MINISTRY OF PUBLIC EDUCATION, COSTA RICA

\footnotetext{
1 Asesor Nacional en Currículum, Consejo Nacional de Enseñanza Superior Universitaria Privada. Ministerio de Educación Pública. Máster en Planificación Curricular, de la Universidad de Costa Rica. Costa Rica. Coordinador Proyecto Sortilegios Educativos.
}

Dirección electrónica: cesartoruno@gmail.com

Ensayo recibido: 25 de agosto, 2014

Enviado a corrección: 4 de diciembre, 2014

Aprobado: 20 de abril, 2015 


\section{Introducción}

El currículum es una estructura educativa que cumple funciones de control (Apple, 1997) que históricamente ha funcionado para la transmisión de un conocimiento oficial (Apple, 1996b) construido, y utilizado por una ideología dominante, es, al mismo tiempo, un escenario para la construcción de la emancipación (Kemmis, 1998), es un problema moral (Gimeno, 1998) y una herramienta vital para mantener o redistribuir las redes de poder en torno al capital cultural y su traducción en la desigual distribución de la riqueza. Como lo escribe Gimeno (2001, p. 40), es un "proyecto selectivo de cultura, cultural, social, política y administrativamente condicionado que rellena la actividad escolar, y que se hace realidad dentro de las condiciones de la escuela tal como se halla configurada".

Por lo tanto, el currículum no es un producto neutral, objetivo ni natural de los procesos sociales-educativos y, mucho menos, exento de conflictos socioculturales y políticoeconómicos propios de la lucha de diversos grupos de presión que trabajan para ampliar su posición (grupo dominante) o redistribuir pesos pedagógicos-sociales (sectores débiles y dominados). El currículum puede definirse como la manifestación explícita de un proyecto cultural, social, político y económico que se estructura contextual e históricamente determinado dentro de la interacción dialéctica Sociedad-Sistema Educativo que, a su vez, genera marcos conceptuales y reguladores para la conformación tangible e intangible del sistema escolar y que, por lo tanto, se configura como un escenario de resistencias al proyecto hegemónico vigente.

Desde su planificación hasta su ejecución y evaluación, los procesos curriculares deben ser reivindicados como procesos pertinentes a nivel técnico, social y moral, comprometidos con los más débiles dentro del sistema (pedagógico, social, económico, cultural, etc.) y espacios vitales para la reflexión sistemática y crítica sobre la cotidianidad curricular. En tal sentido, la selección y la organización de los contenidos como un proceso curricular permite establecer los contenidos como un "espacio para la toma de decisiones en el desarrollo curricular" (Zabalza, 2004, p. 128), que se da dentro estructuras sociales, institucionales y redes de poder (Magendzo, 2008), por lo cual, la selección de contenidos en el currículum prescrito adquiere relevancia por la función sociocultural producto de una estructuración de respuestas a factores académicos, sociales, redes de poder, políticas, ideológicas y subjetividades.

En tal sentido, y como proceso de reflexión-acción para la transformación de estos procesos como construcciones curriculares, el presente ensayo procederá a elaborar una 
breve contextualización de los procesos de reforma de programas de estudio en el Ministerio de Educación, contexto en el cual se elaborará la reflexión teórica de las etapas y procesos sugeridos desde un posicionamiento curricular para, posteriormente, realizar una descripción y explicación de estas etapas dentro del proceso cotidiano de la respectiva comisión de revisión y reforma de programas de estudio, para finalizar con los lineamientos curriculares para la selección y organización de contenidos.

\section{Contexto de las reformas de programas de estudio en el Ministerio de Educación Pública}

El Consejo Superior de Educación es la institución rectora del sistema educativo pre universitario público y privado (en este último caso, con una regulación y acción reducida), con la potestad de revisar y reformar los programas de estudio de las diferentes asignaturas. El proceso para desarrollar una revisión o reforma de algún programa de estudio inicia con la solicitud expresa del Ministro o de la Ministra de Educación para conformar una comisión de revisión o reforma, con una integración que no se encuentra definida en el marco administrativo-legal de la institución por lo que puede variar de 3 a 7 integrantes, liderados por un funcionario nominado directamente por el Ministro o la Ministra, quien toma la atribución de integrar el equipo de trabajo (por la tradición interna, la comisión se integra con un experto externo, asesores regionales o nacionales y docentes) y establece el cronograma de trabajo.

El presente ensayo aportará lineamientos en el marco del trabajo curricular de la comisión de revisión o reforma la cual, en la dinámica institucional, realiza un proceso con las siguientes etapas: análisis del programa vigente, propuesta de ejes estructurantes de la reforma, selección y organización de contenidos, elaboración de estrategias de mediación y evaluación para, finalmente, entregar una propuesta a quien esté al frente del Ministerio, quien a su vez eleva la propuesta al Consejo Superior de Educación, órgano que procede a realizar las consultas públicas con sindicatos, Colegio de Profesores y Licenciados en Ciencias y Letras, Universidades Públicas y otros organismos.

Sistematizadas las observaciones emitidas en la consulta pública, el Consejo Superior de Educación solicita a la comisión la incorporación de los cambios y, recibida la propuesta final con las correcciones, procede a votar a favor, o no, la respectiva propuesta. Todo este proceso se desarrolla en, aproximadamente, 12 meses. 


\section{La selección de contenidos como proceso curricular}

Como ha sido descrito en el apartado anterior, las reformas a los programas de estudio en el Ministerio de Educación Pública de Costa Rica cuentan con una organización administrativa y una serie de etapas diseñadas desde una posición técnica, sin embargo el proceso, por las dinámicas cotidianas o como acción planificada, omite espacios de reflexión, auto evaluación y acción dirigidas a la construcción de un diseño curricular pertinente, relevante y oportuno desde ópticas integrales y críticas. En este sentido, a continuación se esbozarán las características que pueden ser desarrolladas en la selección de los contenidos de los programas de estudio con el objetivo de acercar este proceso a un currículum integral y crítico.

En un proceso de reforma de programa de estudio coherente con los postulados de diseño e implementación curricular, la selección de contenidos debe estar integrada, al menos, por las siguientes fases: a) delimitación de los marcos reguladores generales, b) marcos delimitadores de la selección y organización y, c) aplicación del marco de selección y organización de contenidos. En relación con la primera fase, delimitación de los marcos reguladores generales, se debe explicitar su papel como elemento crucial en la selección de contenidos en tanto, desde una pedagogía crítica, es el escenario de confluencia de las fuentes curriculares, los fundamentos, las estructuras sociales y subjetivas, todo dentro de un marco de relaciones de poder y ampliación-resistencia de un proyecto hegemónico.

La delimitación de los marcos reguladores generales incluye, como primera etapa la formulación ontológica del ser humano-ciudadano a formar y los supuestos epistemológicoshumanistas que guiarán la propuesta, la cual implicará la reflexión crítica de los miembros de la comisión sobre el ideal de ser humano y ciudadano dentro del contexto donde se desarrolla la reforma y, como elemento primario, una coherencia curricular con los fines de la educación costarricense y la política educativa vigente como delimitador de la visión epistemológica y humanista.

Como segunda etapa de la delimitación, resulta necesario la creación del perfil de salida del ciclo y por asignatura, es decir, la formulación ontológica del ser humano y ciudadano implica su operacionalización en la creación de un perfil de salida de ciclo y por asignatura que, para el área de la selección, delimite los conocimientos, sensibilidades, habilidades, destrezas, valores y actitudes que se desean desarrollar. Esta etapa es fundamental en tanto delimitará todos los procesos que se realizarán posteriormente (como 
la selección y organización de contenidos) y ofrece los parámetros para evaluar la coherencia del diseño curricular.

En relación a la segunda fase, marcos delimitadores de la selección y organización, y siguiendo a Zabalza (2004), el primer paso implicará la revisión de la literatura especializada en áreas de la disciplina de la asignatura, currículum (para la comprensión crítica del proceso de reforma y sus implicaciones) y de la evaluación como escenario de mejora de los procedimientos. Así mismo, se deberá realizar una identificación de los contenidos axiales o nudos de ese campo, con la incorporación de expertos en el área disciplinar, ya que: "El experto nos aportará una visión amplia y comprehensiva del área temática, de su endoestructura, ayudándonos a discriminar entre lo fundamental y lo accesorio, y a captar relaciones existentes entre los diversos puntos clave" (Zabalza, 2004, p. 129). Así mismo, se deberá considerar la inclusión de expertos colaboradores provenientes de áreas como filosofía (ética y filosofía), sociología (comportamiento social, estructuras de poder y otros), psicología (procesos de aprendizaje y socioemocionales) u otras que permitan ampliar la reflexión y debate sobre la pertinencia de los contenidos, su organización y sobre el proceso en sí mismo.

Posteriormente, se debe proceder con la selección de contenidos a partir de áreas temáticas generales y la creación de criterios que servirán como redes pedagógicas por las cuales se filtraran los contenidos disciplinares para convertirlos en contenidos didácticos a ser recontextualizados en las aulas. La red o filtro utilizado para seleccionar los contenidos deberá orientarse por criterios que permitan una coherencia curricular entre el perfil, las pertinencias disciplinares y la relevancia para proyectos educativos transversales, por lo cual resulta pertinente los siete criterios sugeridos por Zabalza (2004); en primer lugar el criterio representatividad, en el cual se parte de la premisa de que los contenidos seleccionados representen el área temática o contenido general incluido como referente, a lo cual debe integrarse el criterio de Criterio de ejemplaridad, es decir, contenidos que se consideren básicos para la asignatura y que adquieren importancia en relación a: I) su sentido instrumental "el sujeto podrá utilizarlos para su propio desarrollo cognitivo" (Zabalza, 2004, p.131) y, II) por su sentido lógico, es decir, su capacidad "para estructurar en torno a ellos las distintas nociones de ese campo" (Zabalza, 2004, p. 131).

Como tercer criterio de la selección se establecerá la significación epistemológica, entendida como el reconocimiento vital al respeto de la estructura epistemológica de la disciplina o ciencia, por lo tanto, la selección debe mantener los contenidos claves para el 
desarrollo apropiado del área temática, sin obviar la correlación con el modelo epistemológico definido por la política educativa vigente.

La transferibilidad de los contenidos, como cuarto criterio, se vincula con la capacidad intrínseca de los contenidos aprendidos para ser aplicables en otras circunstancias de la vida y en otras áreas académicas, lo cual es complementado con el criterio de durabilidad, se debe realizar una selección de contenidos de respuesta estructural que puedan servir al estudiantado durante gran parte de su vida, en este caso, como ciudadanos activos de un sistema democrático, ya sea por el contenido en sí o por las habilidades-destrezas y sensibilidades que pueden generar a largo plazo.

El sexto criterio, es la convencionalidad y consenso es decir "recoger aquello con respecto a cuya importancia y validez hay acuerdo en la comunidad escolar" (Zabalza, 2004, p. 131), sin embargo este criterio conlleva una transparencia de procesos para evitar que las subjetividades de la comisión de reforma sean los parámetros para legitimar, o no, una supuesta convencionalidad de un contenido. Por último, Zabalza sugiere el criterio de la especificidad, comprendida como la responsabilidad pedagógica de ofrecer aquellos contenidos que, de otra manera, no serían vistos en otra asignatura o escenario académico, lo cual desde un posicionamiento crítico puede conllevar una reflexión sobre el límite entre lo necesario y aquella acumulación de contenidos que atenta contra los otros criterios.

Además, Hernández (1998) sugiere tres estrategias para descomponer los contenidos que, al mismo tiempo, pueden servir para estructurar la selección de contenidos, siendo relevante para la presente propuesta la asociación libre basada en "la eliminación de todo control, procurando asociar toda la información con todas las posibilidades o enfoques, es decir, trabajando un núcleo temático desde todos los ángulos” (Hernández, 1998, p. 97).

\section{Fundamentos curriculares para la organización de contenidos.}

Posterior a la fase de la selección (construcción de los criterios de selección y determinación de los contenidos que integrarán la propuesta), es necesario desarrollar la organización de los contenidos, momento curricular en el cual, acertadamente, Zabalza (2004, p. 132) sentencia que "el orden en que se presentan los contenidos tiene incidencia en los resultados de aprendizaje".

Los tipos de organización dependen de varios criterios, entre estos "a) naturaleza de la materia; b) del nivel de los alumnos, c) de los recursos disponibles (tiempo, material, lugar, planificación del centro) y d) de la teoría educativa del profesor" (Hernández, 1998, p. 92), 
aunque tales criterios son visualizados para la organización de contenidos en el nivel micro curricular, aportan un insumo para la visualización de la organización de los contenidos en su recontextualización curricular.

En ese sentido, ubicado a nivel micro curricular, Hernández (1998) sugiere el elemento proposional de los contenidos y su definición en relación con su función y forma, para lo cual aporta dos criterios que pueden ser incluidos dentro de un proceso de organización de contenidos en un programa de estudio, estos son: a) distribución vinculado con una manera de organización que agrupa los contenidos por tres criterios, b) enumeración, que distribuye los contenidos sin criterio específico utilizando únicamente una numeración continua en su organización, c) ordenación, situación en la que los contenidos se organizarán por el criterio de magnitud (cantidad, tamaño, tiempo e importancia) y, d) Clasificación, proceso que implica dividir los contenidos por referentes. $\mathrm{O}$, como segundo elemento, la interrelación, es decir, el criterio con base en las relaciones que pueda establecer el contenido, entre estas relaciones están: comparación, causa-consecuencia y circunstancial (propiedades extrínsecas del contenido).

Por su parte Zabalza (2004) propone la organización de contenidos siguiendo una secuenciación que puede ser definida en los términos de Escudero, Area, Bolívar, González, Guarro, Moreno y Santana (1999, p. 53), como "el orden en que vamos a enseñar cada contenido y cuál va a ser su desarrollo". La secuenciación, puede darse siguiendo los criterios de tipo lógico, epistemológico, cognitivo, social, ético y político; no obstante, coinciden con Zabalza en la utilización de un criterio lógico: "En las áreas del ámbito humano y social (geografía, historia, literatura, etc.), este criterio es de difícil aplicación dado que las relaciones lógicas entre sus contenidos son difíciles de establecer y más aún de consensuar" (Escudero et al., 1999, p. 53)

En relación con la secuenciación, Zabalza (2004) propone la secuenciación lineal o compleja como sistema de guía para su estructuración, ellas se subdividen en secuencias simples o lineales que se subdividen en: a) secuencia homogénea (todos los contenidos reciben la misma importancia), b) secuencia heterogénea (los contenidos reciben diferente importancia en el programa de estudio), c) secuencia equidistante (los contenidos reciben el mismo espacio-tiempo concebido a los otros) y d) secuencia no equidistante.

Por su parte, las secuencias complejas se subdividen en: a) Secuencia compleja con alternativas la cual implica que además de la relación relevancia y duración, se ofrece alternativas para que el docente pueda alternar opciones para llegar un contenido más 
general, b) Secuencia compleja con retroactividad en la cual se prevén alteraciones en su abordaje, retrocesos y adelantamientos. Tal secuencia implica un análisis de las características del currículum recontextualizado en las aulas para verificar las posibilidades de tal abordaje, c) Secuencia en espiral en la cual los contenidos se ubican de manera equidistante y con un posible abordaje y análisis idéntico y d) Secuencia convergente, donde los contenidos serán abordados desde diferentes perspectivas, situación que puede ser ejemplificada con los ejes transversales de la educación.

\section{Lineamientos curriculares para la selección y organización de contenidos en los programas de estudio del Ministerio de Educación Pública}

Los siguientes lineamientos curriculares son una propuesta de marcos estructuradores cuyo objetivo es la mejora de los procesos de selección y organización de los contenidos por parte de la comisión de revisión o reforma de los programas de estudio del Ministerio de Educación Pública en sus diferentes asignaturas; además, parten de la premisa de ser una guía que permita la reflexión-acción-reflexión, dentro de la comisión, para diseñar sus propios criterios para seleccionar y organizar los contenidos.

Por lo anterior, la estructura de los lineamientos se desarrollará a partir de los aspectos más generales del proceso, reflexiones iníciales y marco ontológico, para pasar por el área media operacionalizadora de los marcos reguladores y, finalmente, delimitar los criterios curriculares para la selección y la organización de los contenidos.

\subsection{Reflexión inicial}

La labor de la comisión de reforma o revisión de los programas de estudio debe responder a demandas curriculares del proceso dentro de un período de tiempo aproximado de un año, con presiones de elementos externos a la comisión y no necesariamente técnicos como el Consejo Superior de Educación, el Ministro de Educación, las casas editoriales, grupos de presión, las unidades académicas de las universidades y la sociedad civil, a lo cual debe ser sumado las prácticas institucionalizadas que influyen en el hacer cotidiano de la comisión.

En el marco del contexto descrito anteriormente, la comisión deberá iniciar el proceso con la reconceptualización de sus funciones, dejando de lado una visión técnica y limitada a ejercer acríticamente sus roles dentro de la elaboración, o revisión, de un programa de estudio, para dar paso a una autodefinición como intelectuales transformadores (Giroux y 
McLaren, 1998), reconstructores de las dinámicas de sus labores mediante un análisis crítico de las implicaciones de sus acciones, u omisiones, de las funciones, implícitas o no, de los contenidos a seleccionar, las redes de poder y los grupos de presión activamente ligados al proceso y otros fenómenos que enmarcan la creación de productos culturales.

Estas reflexiones, y otras que cuestionen lo normalizado e invisibilizado dentro de la educación como institución cultural, implicarán la reivindicación de la pedagogía crítica como insumo vital para revitalizar las acciones institucionales del Ministerio de Educación Pública en relación con la revisión o la reforma de los programas de estudio, así como lograr una mayor pertinencia sociocultural de sus productos y legitimidad de sus proceso.

La operacionalización de esta reconceptualización de los roles de la comisión, mediante reflexiones críticas, será posible mediante al planteamiento de preguntas generadoras que permitan la apertura de debates y de reflexiones del colectivo, para construir consensos en relación con objetivos, funciones y metas, y que generen interpretaciones comunes sobre el contexto de la reforma, las redes poder y sus influencias, los grupos de presión, la visión ontológica, etc.

Las preguntas generadoras deberán ser construidas en colectivo por los integrantes de la comisión a fin de que sus objetivos no respondan a las subjetividades de un solo miembro de la comisión y deben ser redactadas con una amplitud que les permita incluir grandes temas de la relación Educación y Sociedad, Currículum y posibilidades de transformación para motivar una amplia deliberación mediante la construcción de respuestas, por lo que las preguntas deben ser formuladas con una amplitud tal que impida las respuestas simples (si y no) o que lleven a reduccionismos lógicos; preferiblemente centradas en un eje temático y con una estructura organizativa que vaya de lo más general a lo más específico. Por último, al menos algunas de ellas deben ser planteadas en relación con la estructura en general del currículum con el objetivo de ser analizadas en diferentes momentos del proceso.

Algunas preguntas que podrían plantearse son: ¿En qué macro modelo económico y político se desarrolla la selección y organización de los contenidos?, ¿Cuál es la función (reproductora-productora, generadora de mano de obra-formación sociocultural y otros) que la sociedad asigna o prioriza para la educación? ¿Cuáles son los intereses, explícitos e implícitos, del modelo económico y político en relación con el currículum y el trabajo de la comisión? ¿Cuáles son las redes de poder, explícitas e implícitas, que se manifiestan durante la construcción del diseño curricular y en la selección-organización de los contenidos del currículum prescrito? A nivel de Proceso y Producto, ¿Los integrantes de la comisión son 
técnicos aplicadores o intelectuales transformadores? ¿Cuál es el rol asignado a los docentes dentro del proceso de selección y organización? ¿La selección y la organización de contenidos se desarrollará dentro de procesos y políticas democratizadoras y deliberativas que permitan la participación de todas las voces afectadas, directa e indirectamente, por el diseño curricular propuesto?, ¿Cuál debe ser la pertinencia social, ética y crítica del contenido seleccionado y organizado por la Comisión? ¿El proceso de selección y de organización de contenidos ha priorizado aquellos que sean producto del desarrollo y aplicación de destrezas y habilidades realmente pertinentes dentro del contexto socio histórico?

\subsection{Lo ontológico}

Realizadas las reflexiones críticas iniciales, los integrantes de la comisión enfrentarán la construcción de un marco regulador estratégico para la selección y la organización de contenidos: la visión de ciudadanía y ser humano que debe ser formado por el sistema educativo, así como la proyección de sujeto a formar académicamente.

Dicha construcción no es un producto casual ni una respuesta a las subjetividades de los integrantes de la comisión por el contrario, es un producto complejo que surge de la mezcla de una serie de características del contexto que categorizaremos en los fundamentos curriculares.

Los fundamentos curriculares son una categorización de razonamientos teóricos, disciplinares, sociales, culturales que, estructurados o no, delimitan implícita e explícitamente los procesos curriculares y sustentan los ejes del para qué y por qué enseñar y aprender; entre estos fundamentos están: el sociocultural (puede ser fragmentado en social y cultural), el político, el económico, el psicológico y el filosófico.

Es recomendable la aplicación de preguntas generadoras para clarificar cada fundamento en el proceso de la comisión, como una relación dialéctica, y, al mismo tiempo, producir los objetivos y características del perfil de egresado del sistema educativo en el tercer y cuarto ciclo.

A continuación se expondrán los cuatro fundamentos que integran los lineamientos curriculares, sin embargo la comisión podría decidir establecer más fundamentos con la salvedad teórica de que es poco aconsejable disminuir la cantidad aquí propuesta, esto en relación con la complejidad conceptual de cada asignatura y el estilo de desarrollo operativo de la comisión. 
El primer fundamento curricular es el filosófico, el cual hace referencia a las concepciones, implícitas y explícitas, del ser humano y ciudadanía, que es extraído de los documentos oficiales, como leyes (siendo referente los fines de la educación establecidos en la Ley Fundamental de Educación), reglamentos y programas, de las concepciones socioculturales y políticas de las personas que participan en la selección y en la organización de los contenidos y, por último, del contexto sociopolítico, económico y las redes de poder en las que se construye el diseño curricular.

En relación con la construcción de preguntas generadoras para desarrollar la visión filosófica actual de ser humano y de ciudadanía, se pueden sugerir las siguientes: ¿Cuál es el ideal de ser humano y ciudadanía? ¿Cuáles valores deseamos en ese ser humano? ¿Cuál debe ser la interacción de ese ser humano y ciudadano con la comunidad y el Estado? ¿Cuál es el papel de los conocimientos adquiridos en nuestra asignatura dentro de la interacción ciudadana, social y cultural para el ser humano en formación?

El segundo fundamento curricular es el socio cultural y político, tradicionalmente, estos dos fundamentos se analizarían en individualmente; no obstante, al asumir el fundamento sociocultural, este se interpretará como las demandas sociales y culturales en las que es formado el ciudadano-ser humano y a las cuales tendrá que insertarse y, por su parte, el fundamento político es concebido como las expectativas establecidas para la participación ciudadana en las diferentes estructuras del gobierno del país y la legitimación del régimen político y sus instituciones; en tal sentido, la presente propuesta ha unificado los dos elementos, ya que ambos forjan la creación de parámetros mínimos para la convivencia en las estructuras sociopolíticas del Estado y la comunidad, real e imaginada, en que se desenvolverá cada sujeto.

A nivel sociocultural, se sugiere el planteamiento de las siguientes preguntas: ¿El estudiante es receptor cultural o receptor-constructor cultural? ¿Qué es cultura? ¿Qué es socialización? ¿Cuál es el papel asignado culturalmente a los contenidos de nuestra materia? ¿Cuál debe ser el papel de esos contenidos en la formación del estudiante? ¿Existirá el espacio curricular para el desarrollo de estos contenidos con una clara interrelación con la realidad del estudiantado? ¿Cuáles? ¿Cuál es el papel curricular de estos contenidos para la formación crítica del estudiantado?

Por su parte, a nivel político, desde una pedagogía crítica resulta vital la promoción, el desarrollo y la expansión de un proyecto de ciudadanía y democracia dirigidas a la 
democratización real, la participación activa y crítica, principios de equidad, solidaridad, justicia y transformación.

Como parte de un primer acercamiento, se sugiere las siguientes interrogantes: ¿los contenidos seleccionados fomentan la construcción de una democracia real? ¿Cuál es la proyección de dinámica de aula desarrollada desde el currículum prescrito? ¿Vertical u horizontal? ¿Los contenidos, y su desarrollo, legitiman alguna posición política, económica o cultural? ¿Cómo se asociarán los contenidos seleccionados con el perfil de ser humano establecido en los fines de la educación costarricense? Además, en relación a los ejes transversales y los contenidos que estos exigen para su desarrollo dentro de todas las asignaturas, se pueden plantear las siguientes preguntas: ¿Cuáles son las características mínimas del ciudadano apropiadas al contexto sociocultural? ¿Cuáles son las características mínimas del ciudadano pertinentes desde una visión progresista y humanista? ¿Cuáles instituciones de la democracia costarricense son necesarias reforzar en imaginario colectivo? ¿Cuál es la interacción idealizada dentro del Estado? ¿Cuáles mecanismos reforzarán esa interacción? ¿Cuál es la relación entre la democracia-ciudadanía y los intereses del grupo dominante y los grupos dominados? ¿Qué contenidos promueven la transformación de las relaciones de poder y paradigmas dominantes?

Las anteriores preguntas no tienen un carácter prescriptivo; no obstante, poseen un componente esencial que la comisión deberá retomar, pues radica en el insistente cuestionamiento sobre lo que, normalmente en estos procesos, se da como obvio y, por ende, fácilmente omitido o abarcado en aspectos generales y en corto tiempo podría implicar la omisión o minimización de las complejas dinámicas de sistematizar lo cultural y operacionalizarlo en el currículum prescrito.

El tercer fundamento es el económico, en tanto la construcción del ser humano y la ciudadanía tiene, como referente directo o indirecto, una posición de ese sujeto dentro de la producción económica de la sociedad en la que se desenvuelve.

Al establecer la fundamentación económica dentro de la selección y organización de contenidos, se procura reivindicar los impactos curriculares de posiciones económicas en específico, y los márgenes de acción desde una pedagogía crítica en la formación del sujeto como agente económico activo, iniciando con un análisis crítico de la interacción del papel ciudadano en el modelo económico socialdemócrata dominante en la legislación vigente y la visualización en un modelo neoliberal dominante en el contexto del diseño curricular y la cotidianidad en que se desarrollan. 
Entre las preguntas generadoras para la construcción de ese ciudadano como agente económico, podemos sugerir los siguientes: ¿Cuál es el modelo económico dominante en el país? ¿Cuáles son sus principales características en la cotidianidad sociocultural y productiva? ¿Cuáles son las demandas por parte de los sectores patronales y obreros en relación con la formación del estudiantado? ¿Cuáles son los conocimientos básicos que deberá tener un ciudadano para desenvolverse en el sistema productivo como trabajador y consumidor? ¿Cuáles conocimientos fomentarán una formación crítica de los ciudadanos como trabajadores y consumidores? ¿Ayudarán, los contenidos seleccionados, a la construcción de un nuevo sistema económico más justo, equitativo y solidario? ¿Cuáles contenidos ayudarían a la formación crítica en relación al papel del sistema económico en la vida cotidiana de las personas? ¿Cuáles contenidos fomentarían una visión crítica del actual reparto de la riqueza, tanto sus causas como de los mecanismos de solución?

Por último, el cuarto fundamento curricular es el Psicológico. Este fundamento hace una referencia directa a las teorías psicológicas que guían, implícita y explícitamente, el proceso de selección y de organización de los contenidos, en tanto permite la visualización del sujeto que recontextualizará el currículum (docente) y el sujeto al cual se dirige el diseño curricular que lo transformará en un currículum real; es decir, el estudiante.

En su política educativa, el Ministerio de Educación Pública de Costa Rica ha sido claro en que el constructivismo es la base psicológica de sus procesos educativos, razón por la cual, dentro de las posibles preguntas generadoras que debería plantearse la comisión están: ¿Qué es constructivismo? ¿Cuáles son sus principales características? De esas características, ¿cuáles influyen en la organización de los contenidos? ¿Cómo se organizarán los contenidos respondiendo a los principios constructivistas? Y en ese sentido, ¿la organización de un determinado contenido en un nivel de secundaria responde al nivel cognitivo apropiado para la maximización de las habilidades y destrezas cognitivas esperadas?

Es evidente que, para efectos prácticos en el diseño curricular, el fundamento psicológico asumido en la presente propuesta responde a las inquietudes de la comisión posterior a la selección de los contenidos, es decir, a la organización de estos; no obstante, al menos las primeras tres preguntas sugeridas pueden ser analizadas durante la reflexión inicial de la comisión como generadora de consensos que facilitarían la operacionalización de la organización de contenidos. 
La información obtenida a partir de las preguntas y revisión de documentos para clarificar los fundamentos curriculares, tiene como objetivo la estructuración de un perfil de salida y la obtención de criterios psicológicos para la organización de contenidos, en el primer caso, se procederá a clarificar la operacionalización del perfil de salida.

\subsection{Concreción: perfil de salida}

El perfil de salida constituye la caracterización de habilidades, destrezas, sensibilidades, valores y conocimientos que serán impulsados durante el abordaje de los contenidos, por lo tanto, el perfil de salida cumple una doble función; en primer lugar, como producto explícito de una idealización de ciudadano, y ser humano, planteado por la sociedad y estructurado en el currículum a partir de la reflexión y análisis del contexto mediante la estructuración de los fundamentos curriculares. En segundo lugar, representa un gran estructurador que guiará al proceso educativo, en específico el dado dentro de la asignatura, para delimitar acciones pedagógicas que conlleven a la realización del perfil de salida de cada ciclo.

La creación del perfil de la asignatura o de un ciclo educativo debería ser un complejo proceso democratizador con la interacción de diferentes agentes sociales y con una amplia participación de docentes, estudiantes y otros agentes educativos; sin embargo, la realidad de las reformas y de las revisiones impulsadas por el Ministerio de Educación Pública impiden, para efectos prácticos, la construcción democratizadora del perfil.

En tal sentido, es recomendable la construcción del perfil mediante la utilización de la información construida por los procesos de análisis y reflexión de los fundamentos curriculares y una validación de ese perfil mediante una pequeña consulta a representantes institucionales, agentes sociales y agentes educativos, que podría realizarse por medio encuestas web u otro mecanismo para agilizar el tiempo de recepción de sugerencias.

Para el caso de la elaboración del perfil de salida se requiere una redacción que asuma la forma de un objetivo por cada elemento, con un verbo en infinitivo como palabra inicial de la oración, una extensión corta y centrada en sólo un elemento, pues varios elementos o la ambigüedad en su redacción dificultan su operacionalización por parte de los docentes.

\subsection{De lo ontológico al marco regulador}

Las etapas previas sustentan la conformación de marcos reguladores del proceso de selección y organización de contenidos dentro un espacio académico-profesional pre 
estructurado, el cual inicia con la definición de las áreas disciplinares y temáticas que son necesarias para "extraer" los contenidos académicos que se utilizarán para la concreción de cada objetivo del perfil de salida de la asignatura o el ciclo.

Según sea el caso de la asignatura y la complejidad de contenidos a desarrollar, sus contenidos deben ser asumidos de dos o más áreas disciplinares. Por ejemplo, en el caso de Educación Cívica, por citar una posible asignatura, si un objetivo del perfil de salida establece que el estudiante, como actual y futuro ciudadano, debe "Valorar la democracia como régimen político", tal objetivo puede ser desarrollado desde el Derecho, la Historia y la Sociología, sólo por citar un ejemplo. La definición del área disciplinar, desde el que se elaborará el abordaje de contenidos tendrá una alta influencia en la visión, la criticidad y las perspectivas que puedan generarse en la elaboración de contenidos y su desarrollo en el aula.

Definido el objetivo del perfil y las disciplinas que aportarán los contenidos, es necesario estructurar las áreas temáticas que se abordarán dentro del perfil de salida en tal sentido, y continuando con nuestro ejemplo del perfil, se podrían estructurar las siguientes áreas temáticas: Perfil: "Valorar la democracia como régimen político". Áreas disciplinares: Historia, Ciencias Políticas y Sociología. Áreas temáticas Historia: a. Evolución del concepto de Democracia desde Grecia hasta nuestros días. b. Construcción de la democracia durante y después del siglo de las luces. Derecho: a. Marco legal en democracia Derechos y deberes en la democracia. Sociología: a. Cultura Democrática como forma de convivencia. b. Formas de participación en una sociedad democrática.

Es necesario hacer explícita la preocupación porque las áreas temáticas sean asumidas desde una posición crítica coherente con el perfil de currículo propuesto, esto por cuanto sería una contradicción la implementación de áreas temáticas con una visión y contenidos acríticos, legitimadores del discurso oficial y reproductores de desigualdades.

Definidas las áreas temáticas y su relación con ejes transversales, es necesario definir los elementos conceptuales que integrarán las áreas temáticas, dichos elementos no pueden ser tan específicos que impidan la selección de contenidos, o que se vuelva un contenido en sí, ni tan amplio como para no cumplir su tarea de seleccionar los contenidos apropiados y pertinentes de los que no.

Para la definición de estos elementos, es prudente, en primer lugar, una revisión bibliográfica, en tanto, toda área temática tiene una complejidad de informaciones que es preciso abordar, es claro que en las dinámicas de una comisión de reforma o revisión de 
programas de estudio las posibilidades de realizar una revisión exhaustiva son limitadas, una consulta con expertos y la experiencia de los integrantes de la comisión como docentes, aunque esto último puede representar severos sesgos en la selección y organización de contenidos y, en general, en todo el proceso curricular, por lo que el uso de la experiencia como delimitador de elementos debe utilizarse de manera proporcional y, preferiblemente, dirigidos a casos muy específicos en los cuales la revisión bibliográfica y el criterio de expertos no hayan podido clarificar su construcción.

\subsection{Criterios de selección y organización de contenidos}

La selección y organización de contenidos, desde una pedagogía crítica, debe priorizarse como un proceso demarcado por la formación y la aplicación de principios que guiarán el proceso, así como la creación de criterios curriculares. Para seleccionar y organizar los contenidos, se deben establecer criterios que respondan tanto a elementos críticos, que permitan la reflexión sobre su función dentro de un proyecto cultural, como a criterios técnicos que permitan la verificación de principios curriculares elementales.

Asumir la Pedagogía Crítica como rama de reflexión y acción dentro de los procesos de selección y organización de los contenidos es, ante todo, una apropiación de principios que guiarán los procesos, tanto en la acción directa de los participantes como en los mecanismos de regulación y construcción de la selección y organización.

En tal sentido, José Gimeno Sacristán (2001) establece una sistematización y estructuración de principios destinados a dar legitimidad a los contenidos y posibilitar su organización. El primer principio es la claridad sobre la naturaleza humana, del sujeto y la serie de necesidades que deben satisfacerse para lograr su pleno desarrollo y para una buena relación con la sociedad. A ello es necesario agregar la interpretación de Apple (1996b) sobre el rol del sistema educativo en la transmisión, reproducción y consolidación del conocimiento oficial y las posibilidades de resistencia a este proyecto hegemónico.

Como segundo principio se establece la clarificación de los contenidos justificados por su universalidad de hecho, por su utilidad instrumental y los necesarios para el entendimiento y desarrollo de una sociedad determinada. Además, hay contenidos que son necesarios por ser una derivación de la conceptualización, y defensa, de una sociedad democrática y una ciudadanía plural, que están sustentados en aspectos como: Respeto a la diversidad étnica (resulta necesario ampliar este requisito al reconocimiento y respeto de la diversidad en todos los extremos de sus manifestaciones, sean de origen religioso, sexual, 
de clase y otros), Valentía Cívica "para alzar la voz por causas justas, por la libre opinión" (Gimeno, 2001, p. 250), Solidaridad, Justicia, Prudencia y Participación.

Es necesario agregar, como tercer principio, la necesidad de que los contenidos sean evaluados desde sus potencialidades para romper con una pedagogía engañosa, entendida esta como aquella "diseñada para impartir... desde el principio, una información falsa y unas creencias que han sido transmitidas de generación en generación" (Macedo, 1997, p. 132) y, en términos de Giroux (2006) que fomenten una memoria peligrosa, es decir, aquella reconstrucción histórica de la lucha histórica de los más débiles, la construcción social de logros, la visibilización de las injusticias y de las redes de poder, entre otros.

Como cuarto principio se establece el impulso de un marco curricular, en este caso de selección y organización, plural que rechace la interpretación monocultural de los fenómenos sociales. Esto implica, "estimular el interés activo por 'otros' que provienen o pertenecen a otras culturas" (Gimeno, 2001, p. 250). Además, como sugiere Apple (1996b, p. 85) se debe evitar la "tradición selectiva en la que sólo se convierte en conocimiento oficial el conocimiento de determinados grupos". Lo cual nos lleva a un quinto principio, fomentar una cultura común no implica homogeneización cultural, ya que se deben de analizar y evaluar los intercambios culturales (importante dentro de un proceso de globalización) de manera crítica.

Lo anterior se relaciona con el sexto principio, visualizar que la igualdad debe ser un eje elemental "pensando la igualdad desde parámetros culturales, de género, clase social, etc" (Gimeno, 2001, p. 250). Este es un principio que debe ser un marco tanto para el proceso de selección, entiéndase la inclusión horizontal de diversos actores del proceso, y como regulador del producto, lo cual se encuentra vinculado con el séptimo criterio, a saber; desarrollar una cultura para explicar el orden vigente, asumiendo que "la educación es la fuerza fundamental para dar esa conciencia reflexiva sobre el orden del mundo vigente (también sobres sus 'desordenes'), con una mirada crítica acerca de lo que significa" (Gimeno, 2001, p. 253), principio compartido por Apple (1996a, p. 29) y profundizado al establecer que "El trabajo educativo que no está conectado de modo fundamental con una comprensión profunda de estas realidades... corre el riesgo de perder su alma".

El último principio es fomentar una educación reflexiva y la identidad cultural, en términos de Apple (1996a), comprometida con el análisis de la política cultural de la educación y capaz de develar el papel ideológico del sistema educativo (Kemmis, 1998) y 
donde se reconozca "nuestra tarea como educadores con espíritu crítico y democrático es una tarea política" (Apple, 1996b, p. 81)

Además, siguiendo a Freire (1997, p. 21), es necesario realizar una interpretación crítica de la aplicación de estos principios, ya que las personas integrantes de la Comisión de reforma deben asumir que "La mejora de la calidad de la educación implica la formación permanente de los educadores. Y la formación permanente consiste en la práctica de analizar la práctica" situación que se traduciría en un análisis sistemático y crítico de las acciones realizadas por la Comisión para asegurar no sólo una selección y organización objetiva, crítica y pertinente sino, además, asegurar que su proceso sea inclusivo, igualitario y democrático.

Para operacionalizar estos principios, es pertinente que las personas integrantes de la comisión vuelvan a repetir la reflexión inicial y sus preguntas generadoras, buscando la creación de nuevas preguntas que respondan a los ejes de los principios anteriormente expuestos y que permitan una deliberación amplia y transparente a lo interno de la Comisión como proceso vital previo al ingreso de la selección y organización de contenidos como proceso técnico con criterios curriculares.

Desde la perspectiva curricular sobre la selección de contenidos, siguiendo a Hernández (1998) y Zabalza (2004), el presente ensayo propone dos grandes criterios de selección: a) Asociación Libre y b) Proposional y Criterio temático.

En el caso de la Asociación Libre, se establece con base en la idea de núcleos temáticos, lo cual genera una gran flexibilidad y, por ende, la anulación de criterios sistematizados. Esta asociación debe ser estructurada con núcleos temáticos y ofrecer lineamientos que garanticen una discusión pertinente y democratizadora que facilite la construcción del consenso sobre los contenidos seleccionados.

La Asociación Libre daría un nombre técnico a la forma de seleccionar contenidos construida en la cotidianidad de los procesos de selección de contenidos del Ministerio de Educación (Toruño, 2011) pero con la salvedad, en los procesos dentro del MEP, de un manejo disciplinar y técnico curricular mucho más débil de lo deseable para un proceso de tan alta complejidad, por tal motivo no es recomendable desde una posición pro sistematización y estructuración del proceso.

Por lo anterior, asumimos como criterios curriculares fundamentales el Proposicionaltemático, en el caso del criterio proposional el contenido se define en relación a su función y forma, analizando el aprendizaje como un proceso dinámico y activo en construcción 
compleja. Hernández (1998, p. 100) y, en el caso temático, se define en relación con la división académica de asignaturas, materias, áreas u otra división construida por la disciplina.

Además el criterio Proposional se subdividirá en los subcriterios de: a) Especificidad (son contenidos que sólo podrán ser abordados en la materia, es decir, Educación Cívica), b) Durabilidad (el contenido responderá a demandas de mediano y largo plazo y no únicamente a necesidades inmediatas del contexto) y c) Convencionalidad y consenso (el contenido responde a un consenso o convencionalidad de la comunidad, sea nacional o internacional, que legitimen la importancia de su inclusión)

Por su parte, el criterio Temático se subdivide en los siguientes subcriterios: a) Criterio representatividad (se parte de la premisa de que los contenidos seleccionados representen el área temática o contenido general incluido como referente), b) Criterio de ejemplaridad (se apuesta por contenidos que se consideren básicos para la asignatura), que puede dividirse en ejemplaridad instrumental, ejemplaridad lógica, transferibilidad, integrada por contenidos, y c) Significación epistemológica (los contenidos responden a la lógica epistemológica de las diferentes disciplinas, por lo que representan contenidos claves para el desarrollo del área disciplinar en el aula)

Como puede apreciarse, se establecen 10 criterios para justificar la inclusión o exclusión de un contenido dentro de un área temática, en tanto tales criterios permiten un margen de acción dentro de la Comisión de Reforma de los Programas de Estudio para generar adaptaciones, discusiones y productos con una estructura base y, en segundo lugar, como respuesta a las necesidades operativas de los integrantes de la Comisión para encontrarse con una cantidad de criterios que no entorpezcan sus labores en extendidos procesos técnicos.

Posterior a la selección de contenidos, la comisión enfrenta la tarea curricular de organizarlos en los programas de estudio, situación ante la cual se podría dejar al azar; sin embargo, "en el caso de que el aprendizaje de unos elementos facilite el aprendizaje posterior de otros, entonces el establecimiento de un orden cuidadoso en los aprendizajes será preferible a secuencias aleatorias" (Coll, 1991, p. 68), además, el autor rescata las propuestas de Ausubel basada en la secuencia de aprendizaje de acuerdo con los principios cognitivos que rigen la formación y el desarrollo cognitivo.

Por lo anterior, Coll (1991, p. 75) sugiere que "el primer paso consiste en identificar los elementos fundamentales del contenido y en organizarlos en un esquema jerárquico y 
relacional, en torno a los elementos que tengan la máxima generalidad y que puedan integrar el mayor número posible de elementos restantes" y, además, establece las siguientes preguntas para lograr tal organización

¿cuáles son las preguntas claves a las que responde el conocimiento de la disciplina en cuestión?, ¿cuáles son los conceptos clave? ¿qué métodos de investigación utiliza para generar el conocimiento? ¿cuáles son las afirmaciones principales que formula en respuesta a las preguntas clave?; y por último, ¿qué juicios de valor hace intervenir? (Coll, 1991, p. 75)

El segundo paso consiste en ordenarlos según los criterios de la organización psicológica del conocimiento, establecidos por Novak (1982, citado por Coll, 1991, pp. 7577), estos son:

a)Todos los alumnos pueden aprender significativamente un contenido en tanto dispongan de los conceptos relevantes en su estructura cognoscitiva para incluirlos, b) El contenido y los conceptos deben organizarse de lo más general a lo más específico y c) Se deben de manifestar las relaciones entre el contenido más general y el específico

La propuesta de Coll (1991) tiene como objetivo de la organización que los elementos en secuencias vayan de lo más general a lo específico o de lo más simple a lo más complejo, para lo cual establece criterios de secuenciación, interpretando secuencia como "el orden en que vamos a enseñar cada contenido y cuál va a ser su desarrollo" (Escudero et al., 1999)

El primer criterio es la secuenciación interciclos, en el cual se establecen una jerarquía de habilidades, destrezas, posibilidades de acción, capacidades y conocimientos que debe desarrollar el estudiantado (perfil de Salida) tanto de los Objetivos Generales del Ciclo (perfil de Salida General) y los Objetivos Generales del Área (perfil de salida de Educación Cívica). Es importante resaltar que el autor reconoce la ausencia de criterios para establecer la jerarquía ante lo cual sugiere el uso de aproximaciones sucesivas basadas en preguntas generadoras cuyas respuestas permitan la generación de la jerarquía.

Como segundo criterio se sugiere la secuenciación interniveles, partiendo del reconocimiento de los niveles como unidades funcionales de organización para el sistema escolar por lo que los contenidos pueden ser organizados específicamente para ellos (objetivos de nivel) sin obviar su interacción con niveles previos y superiores. Por último, 
existe la secuenciación intraniveles: se establece una jerarquía de contenidos dentro de cada nivel, cuya distribución final tomará forma bajo el concepto de Unidades Didácticas.

A nivel más específico, Coll (1991) propone una a secuenciación de conceptos en donde organizan de los conceptos fundamentales y representativos (generales) y se inicia una introducción, progresiva, de los conceptos más específicos, una secuenciación de principios en la cual se inicia con los principios más simples y fundamentales de las estructuras teóricas para avanzar, progresivamente, a los más específicos, complejos y contextualizados; una secuenciación de procedimientos estructurada a partir de los procedimientos más simples a los más específicos y complejos y la secuenciación de hechos, en la cual se organizan los hechos de tal forma que primero se aborden más representativos y próximos para el estudiantado y, posteriormente, se introducen hechos más singulares y alejados de la experiencia cotidiana del estudiante.

En relación con la secuenciación propuesta por Zabalza (2004), y como complemento a la sugerida por Coll, es pertinente una secuencia simple o lineal (mezcla según la relevancia y duración) heterogénea no equidistante, es decir, donde los contenidos reciben diferente importancia en el programa de estudio sin el mismo espacio-tiempo programado, en tanto habrá contenidos de mayor importancia que requieran mayor espacio-tiempo en el abordaje a nivel micro curricular.

\section{Consideraciones finales}

El currículum es un complejo entramado de intereses académicos, sociales, económicos, políticos y culturales que se desarrolla en un escenario de proyectos hegemónicos y resistencias al mismo. Por lo tanto, cada una de las etapas de la concepción, implementación y evaluación de un currículum deben implicar procesos de reflexión, investigación y toma de decisiones que fomenten una comprensión integral de este, sus implicaciones y posibilidades de mejora.

En el caso del presente ensayo, se ha abordado la selección y organización de contenidos como una etapa del diseño curricular del sistema educativo presentado al personal docente, denominada para el caso costarricense como Programas de Estudio, para lo cual se ha realizado la respectiva conceptualización de cada etapa, para estructurar y sistematizar. Esta etapa es una necesidad vital dentro del Ministerio de Educación Pública. Ya sea desde un posición tecnicista que reivindique exclusivamente la importancia de validar y legitimar el producto, como desde una posición deliberativa que responda a las 
necesidades de mejorar los procesos e incorporar otras voces, así como, por supuesto, desde una pedagogía crítica demandante de un posicionamiento y operacionalización del sistema educativo como institución cultural con escenarios de acción cotidiana que deben ser reformulados críticamente.

A partir de la concepción desde una pedagogía crítica, se han establecido una serie de criterios para la selección y organización de contenidos con el objetivo de construir un proceso reflexivo, democratizador y dialogante que favorezca la construcción de programas de estudio enmarcados en posiciones críticas y humanísticas. Además, se ha realizado una introducción de la pedagogía crítica en los procesos de selección y organización de tal forma que mantenga la coherencia con la corriente pedagógica pero, al mismo tiempo, sea aplicable dentro de la realidad institucional del MEP, permitiendo una primera ruptura dentro de un modelo curricular oficial.

Por tanto, la propuesta esbozada en el presente ensayo pretende contribuir a la mejora curricular en los procesos de selección y organización y, paralelamente, fomentar el debate académico sobre el quehacer curricular desarrollado por el Ministerio de Educación Pública y sus impactos educativos, sociales, políticos y económicos, desde una perspectiva crítica. En este último apartado resulta necesario señalar que existe la necesidad de estudios y deliberaciones sobre la inclusión de la pedagogía crítica en el diseño curricular del Ministerio de Educación Pública en aspectos como la influencia de grupos hegemónicos, el proyecto político dominante y su influencia en la estructura educativa, el perfil de ser humano a formar desde una posición crítica, la democratización de la evaluación y la transformación de la contextualización curricular para construir espacios de aprendizaje dinámicos, democráticos y humanistas.

\section{REFERENCIAS}

Apple, Michael. (1997). Política cultural y educación. España: Morata.

Apple, Michael. (1996a). Educación y poder. España: Paidós.

Apple, Michael. (1996b). El conocimiento oficial. La educación democrática en una era conservadora. España: Paidós.

Coll, César. (1991). Psicología y currículum. España: Paidós.

Escudero, Juan Manuel, Area, Manuel, Bolívar, Antonio, González, María Teresa, Guarro, Amador, Moreno, Juna Manuel y Santana, Pablo. (1999). Diseño, desarrollo e innovación del currículum. España: Síntesis. 
Freire, Paulo. (1997). Educación y participación comunitaria. En Manuel Castells, Ramón Flecha, Paulo Freire, Herny Giroux, Donaldo Macedo y Paul Willis, Nuevas perspectivas críticas en educación (pp. 83-96). España: Paidós.

Gimeno, José. (2001). Educar y convivir en la cultura global. Las exigencias de la ciudadanía. España: Morata.

Gimeno, José. (1998). Poderes inestables en educación. España: Morata. S.A.

Giroux, Henry y McLaren, Peter. (1998). Sociedad, cultura y educación. España: Miño y Dávila.

Giroux, Henry. (2006). La escuela y la lucha por la ciudadanía. Pedagogía crítica de la época moderna. México: Siglo XXI.

Giroux, Henry; McLaren, Peter. (1998). Sociedad, cultura y educación. España: Miño y Dávila.

Hernández, Pedro. (1998). Diseñar y enseñar. Teoría y técnicas de la programación del proyecto docente. España: Narcea.

Kemmis, Stephen. (1998). El currículum: más allá de la teoría de la reproducción. España: Morata.

Macedo, Donaldo. (1997). Nuestra cultura común: una pedagogía engañosa. En Manuel Castells, Ramón Flecha, Paulo Freire, Herny Giroux, Donaldo Macedo y Paul Willis, Nuevas perspectivas críticas en educación (pp. 126-165). España: Paidós.

Magendzo, Abraham. (2008). Dilemas del currículum y la pedagogía. Analizando la reforma curricular desde una perspectiva crítica. Chile: LOM.

Toruño, César. (2011). La configuración de la ciudadanía en los programas de estudio de Educación Cívica al iniciar el siglo XX: el caso del elemento político. Actualidades Investigativas en Educación, 11(2), 1-25. Recuperado de http://revista.inie.ucr.ac.cr/uploads/tx magazine/configuracion-ciudadania-programasestudio-educacion-civica-toruno.pdf

Zabalza, Miguel. (2004). Diseño y desarrollo curricular. España: Narcea. 